J. Appl. Numer. Optim. 3 (2021), No. 1, pp. 197-210

Available online at http://jano.biemdas.com

https://doi.org/10.23952/jano.3.2021.1.11

\title{
A WEAK CONVERGENCE THEOREM FOR RELATIVELY NONEXPANSIVE MAPPINGS AND MAXIMAL MONOTONE OPERATORS IN A BANACH SPACE
}

\author{
WATARU TAKAHASHI
}

\begin{abstract}
Research Center for Interneural Computing, China Medical University Hospital, China Medical University, Taichung 40447, Taiwan
\end{abstract}

Keio Research and Education Center for Natural Sciences, Keio University, Yokohama 223-8521, Japan

Department of Mathematical and Computing Sciences, Tokyo Institute of Technology, Tokyo 152-8552, Japan

\begin{abstract}
In this paper, using the idea of Mann's iteration, we prove a weak convergence theorem for finding a common element of the fixed point sets of two relatively nonexpansive mappings and the zero point set of a maximal monotone operator in a Banach space. We apply this result to get well-known and new weak convergence theorems which are connected with relatively nonexpansive mappings and maximal monotone operators in Hilbert spaces and in Banach spaces.
\end{abstract}

Keywords. Relatively nonexpansive mapping; Generalized projection; Maximal monotone operator; Generalized resolvent; Fixed point.

\section{INTRODUCTION}

Let $E$ be a Banach space and let $C$ be a nonempty, closed and convex subset of $E$. Let $T$ be a mapping of $C$ into $E$. We denote the set of fixed points of $T$ by $F(T)$. A mapping $T: C \rightarrow E$ is called demiclosed if for a sequence $\left\{x_{n}\right\}$ in $C$ such that $\left\{x_{n}\right\}$ converges weakly to a point $p$ and $x_{n}-T x_{n} \rightarrow 0, p=T p$ holds.

Assume that $E$ is a smooth Banach space and $C$ is a nonempty, closed and convex subset of $E$. A mapping $T: C \rightarrow E$ is called relatively nonexpansive [1] if $F(T) \neq \emptyset$, it is demiclosed and

$$
\phi(z, T x) \leq \phi(z, x), \quad \forall x \in C, z \in F(T),
$$

where $\phi(x, y)=\|x\|^{2}-2\langle x, J y\rangle+\|y\|^{2}$ for all $x, y \in E$ and $J$ is the duality mapping of $E$.

In 1953, Mann [2] introduced the following iteration process. Let $T: C \rightarrow C$ be a nonexpansive mapping, that is, $\|T x-T y\| \leq\|x-y\|$ for all $x, y \in C$. For an initial guess $x_{1} \in C$, an iteration process $\left\{x_{n}\right\}$ is defined recursively by

$$
x_{n+1}=\alpha_{n} x_{n}+\left(1-\alpha_{n}\right) T x_{n}, \quad \forall n \in \mathbb{N},
$$

where $\left\{\alpha_{n}\right\}$ is a sequence in [0,1]. Later, Reich [3] discussed Mann's iteration process in a uniformly convex Banach space with a Fréchet differentiable norm and obtained that the sequence $\left\{x_{n}\right\}$ converges weakly to a fixed point of $T$ under some conditions. On the other

E-mail addresses: wataru@is.titech.ac.jp; wataru@a00.itscom.net.

Received September 4, 2020; Accepted October 20, 2020.

(C)2021 Journal of Applied and Numerical Optimization 
hand, Matsushita and Takahashi [4] proved a weak convergence theorem under Mann's iteration process for relatively nonexpansive mappings in a smooth and uniformly convex Banach space.

In this paper, using the idea of Mann's iteration, we prove a weak convergence theorem for finding a common element of the fixed point sets of two relatively nonexpansive mappings and the zero point set of a maximal monotone operator in a Banach space. We apply this result to get well-known and new weak convergence theorems which are connected with relatively nonexpansive mappings and maximal monotone operators in Hilbert spaces and in Banach spaces

\section{PRELIMINARIES}

We denote by $\mathbb{N}$ the set of positive integers and by $\mathbb{R}$ the set of real numbers. Let $H$ be a real Hilbert space with inner product $\langle\cdot, \cdot\rangle$ and norm $\|\cdot\|$, respectively. Let $C$ be a nonempty, closed and convex subset of a Hilbert space $H$. The nearest point projection of $H$ onto $C$ is denoted by $P_{C}$, that is, $\left\|x-P_{C} x\right\| \leq\|x-y\|$ for all $x \in H$ and $y \in C$. Such $P_{C}$ is called the metric projection of $H$ onto $C$. We know that the metric projection $P_{C}$ is firmly nonexpansive, i.e.,

$$
\left\|P_{C} x-P_{C} y\right\|^{2} \leq\left\langle P_{C} x-P_{C} y, x-y\right\rangle
$$

for all $x, y \in H$. Furthermore, $\left\langle x-P_{C} x, y-P_{C} x\right\rangle \leq 0$ holds for all $x \in H$ and $y \in C$; see [5].

Let $E$ be a real Banach space with norm $\|\cdot\|$ and let $E^{*}$ be the dual space of $E$. We denote the value of $y^{*} \in E^{*}$ at $x \in E$ by $\left\langle x, y^{*}\right\rangle$. When $\left\{x_{n}\right\}$ is a sequence in $E$, we denote the strong convergence of $\left\{x_{n}\right\}$ to $x \in E$ by $x_{n} \rightarrow x$ and the weak convergence by $x_{n} \rightarrow x$. The modulus $\delta_{E}$ of convexity of $E$ is defined by

$$
\delta_{E}(\varepsilon)=\inf \left\{1-\frac{\|x+y\|}{2}:\|x\| \leq 1,\|y\| \leq 1,\|x-y\| \geq \varepsilon\right\}
$$

for every $\varepsilon$ with $0 \leq \varepsilon \leq 2$. A Banach space $E$ is said to be uniformly convex if $\delta_{E}(\varepsilon)>0$ for every $\varepsilon>0$. A uniformly convex Banach space is strictly convex and reflexive. The duality mapping $J_{E}$ from $E$ into $2^{E^{*}}$ is defined by

$$
J_{E} x=\left\{x^{*} \in E^{*}:\left\langle x, x^{*}\right\rangle=\|x\|^{2}=\left\|x^{*}\right\|^{2}\right\}
$$

for every $x \in E$. We also denote $J_{E}$ by $J$ simply. Let $U=\{x \in E:\|x\|=1\}$. The norm of $E$ is said to be Gâteaux differentiable if for each $x, y \in U$, the limit

$$
\lim _{t \rightarrow 0} \frac{\|x+t y\|-\|x\|}{t}
$$

exists. In this case, $E$ is called smooth. We know that $E$ is smooth if and only if $J$ is a singlevalued mapping of $E$ into $E^{*}$. The norm of $E$ is said to be Fréchet differentiable if for each $x \in U$, the limit (2.2) is attained uniformly for $y \in U$. The norm of $E$ is said to be uniformly smooth if the limit (2.2) is attained uniformly for $x, y \in U$. If $E$ is uniformly smooth, then $J$ is uniformly norm-to-norm continuous on each bounded subset of $E$. We also know that $E$ is reflexive if and only if $J$ is surjective, and $E$ is strictly convex if and only if $J$ is one-to-one. Therefore, if $E$ is a smooth, strictly convex and reflexive Banach space, then $J$ is a single-valued bijection and in this case, the inverse mapping $J^{-1}$ coincides with the duality mapping $J_{*}$ on $E^{*}$. For more details, see $[6,7]$. We also know the following result.

Lemma 2.1 ([6]). Let $E$ be a smooth Banach space and let $J$ be the duality mapping on $E$. Then, $\langle x-y, J x-J y\rangle \geq 0$ for all $x, y \in E$. Furthermore, if $E$ is strictly convex and $\langle x-y, J x-J y\rangle=0$, then $x=y$. 
Let $E$ be a smooth Banach space. The function $\phi: E \times E \rightarrow(-\infty, \infty)$ is defined by

$$
\phi(x, y)=\|x\|^{2}-2\langle x, J y\rangle+\|y\|^{2}
$$

for $x, y \in E$, where $J$ is the duality mapping of $E$; see $[8,9]$. We have from the definition of $\phi$ that

$$
\phi(x, y)=\phi(x, z)+\phi(z, y)+2\langle x-z, J z-J y\rangle
$$

for all $x, y, z \in E$. From $(\|x\|-\|y\|)^{2} \leq \phi(x, y)$ for all $x, y \in E$, we can see that $\phi(x, y) \geq 0$. Furthermore, we can obtain the following equality:

$$
2\langle x-y, J z-J w\rangle=\phi(x, w)+\phi(y, z)-\phi(x, z)-\phi(y, w)
$$

for $x, y, z, w \in E$. If $E$ is additionally assumed to be strictly convex, then from Lemma 2.1 we have

$$
\phi(x, y)=0 \Longleftrightarrow x=y .
$$

Let $E$ be a smooth, strictly convex and reflexive Banach space. Let $\phi_{*}: E^{*} \times E^{*} \rightarrow(-\infty, \infty)$ be the function defined by

$$
\phi_{*}\left(x^{*}, y^{*}\right)=\left\|x^{*}\right\|^{2}-2\left\langle J^{-1} y^{*}, x^{*}\right\rangle+\left\|y^{*}\right\|^{2}
$$

for all $x^{*}, y^{*} \in E^{*}$, where $J$ is the duality mapping of $E$. It is easy to see that

$$
\phi_{*}(J y, J x)=\phi(x, y)
$$

for all $x, y \in E$. The following lemma which was by Kamimura and Takahashi [9] is well-known.

Lemma 2.2 ([9]). Let $E$ be a smooth and uniformly convex Banach space. Let $\left\{x_{n}\right\}$ and $\left\{y_{n}\right\}$ be two sequences in space $E$ such that either sequence $\left\{x_{n}\right\}$ or sequence $\left\{y_{n}\right\}$ is bounded. If $\lim _{n \rightarrow \infty} \phi\left(x_{n}, y_{n}\right)=0$, then $\lim _{n \rightarrow \infty}\left\|x_{n}-y_{n}\right\|=0$.

The following lemmas are in $\mathrm{Xu}$ [10] and Kamimura and Takahashi [9].

Lemma 2.3 ([10]). Let $E$ be a uniformly convex Banach space and let $r>0$. Then there exists a strictly increasing, continuous and convex function $g:[0, \infty) \rightarrow[0, \infty)$ such that $g(0)=0$ and

$$
\|\lambda x+(1-\lambda) y\|^{2} \leq \lambda\|x\|^{2}+(1-\lambda)\|y\|^{2}-\lambda(1-\lambda) g(\|x-y\|)
$$

for all $x, y \in B_{r}$ and $\lambda$ with $0 \leq \lambda \leq 1$, where $B_{r}=\{z \in E:\|z\| \leq r\}$.

Lemma 2.4 ([9]). Let $E$ be a smooth and uniformly convex Banach space and let $r>0$. Then there exists a strictly increasing, continuous and convex function $g:[0,2 r] \rightarrow \mathbb{R}$ such that $g(0)=$ 0 and

$$
g(\|x-y\|) \leq \phi(x, y)
$$

for all $x, y \in B_{r}$, where $B_{r}=\{z \in E:\|z\| \leq r\}$.

Let $C$ be a nonempty, closed and convex subset of a strictly convex and reflexive Banach space $E$. For any $x \in E$, we also know that there exists a unique element $z \in C$ such that

$$
\phi(z, x)=\min _{y \in C} \phi(y, x) .
$$

The mapping $\Pi_{C}: E \rightarrow C$ defined by $z=\Pi_{C} x$ is called the generalized projection of $E$ onto $C$. We know the following result. 
Lemma 2.5 ([8, 9]). Let $E$ be a smooth, strictly convex and reflexive Banach space. Let $C$ be a nonempty, closed and convex subset of $E$ and let $x \in E$ and $z \in C$. Then, the following conditions are equivalent:

(1) $z=\Pi_{C} x$

(2) $\langle z-y, J x-J z\rangle \geq 0, \quad \forall y \in C$.

Let $E$ be a Banach space and let $B$ be a mapping of of $E$ into $2^{E^{*}}$. A multi-valued mapping $B$ on $E$ is said to be monotone if $\left\langle x-y, u^{*}-v^{*}\right\rangle \geq 0$ for all $u^{*} \in B x$, and $v^{*} \in B y$. A monotone operator $B$ on $E$ is said to be maximal if its graph is not properly contained in the graph of any other monotone operator on $E$. The following theorem is due to Browder [11]; see also [7, Theorem 3.5.4].

Theorem 2.1 ([11]). Let $E$ be a uniformly convex and smooth Banach space and let $J$ be the duality mapping of $E$ into $E^{*}$. Let $B$ be a monotone operator of $E$ into $2^{E^{*}}$. Then $B$ is maximal if and only if, for any $r>0$,

$$
R(J+r B)=E^{*},
$$

where $R(J+r B)$ is the range of $J+r B$.

Let $E$ be a uniformly convex Banach space with a Gâteaux differentiable norm and let $B$ be a maximal monotone operator of $E$ into $2^{E^{*}}$. The set of null points of a maximal monotone operator $B$ is defined by $B^{-1} 0=\{z \in E: 0 \in B z\}$. We know that $B^{-1} 0$ is closed and convex; see [7].

For all $x \in E$ and $r>0$, we also consider the following equation

$$
J x \in J x_{r}+r B x_{r} .
$$

This equation has a unique solution $x_{r}$; see [12]. We define $Q_{r}$ by $x_{r}=Q_{r} x$. Such a $Q_{r}$ is called the generalized resolvent of $B$. For $r>0$, the Yosida approximation $B_{r}: E \rightarrow E^{*}$ is defined by

$$
B_{r} x=\frac{J x-J Q_{r} x}{r}, \quad \forall x \in E .
$$

When the Banach space is a Hilbert space, we have that the generalized resolvent $Q_{r}$ is called the resolvent of $B$ simply. We know the following result.

Lemma 2.6 ([12]). Let $E$ be a uniformly convex and smooth Banach space and let $B \subset E \times E^{*}$ be a maximal monotone operator. Let $r>0$ and let $Q_{r}$ and $B_{r}$ be the generalized resolvent and the Yosida approximation of $B$, respectively. Then, the following hold:

(1) $\phi\left(u, Q_{r} x\right)+\phi\left(Q_{r} x, x\right) \leq \phi(u, x), \quad \forall x \in E, u \in B^{-1} 0$;

(2) $\left(Q_{r} x, B_{r} x\right) \in B, \quad \forall x \in E$;

(3) $F\left(Q_{r}\right)=B^{-1} 0$.

\section{WEAK CONVERGENCE THEOREM}

In this section, we prove a weak convergence theorem of Mann's type iteration for two relatively nonexpansive mappings and maximal monotone operators in a Banach space. The following lemma was proved by Matsushita and Takahashi [1]. 
Lemma 3.1 ([1]). Let $E$ be a smooth, strictly convex and reflexive Banach space and let $C$ be a nonempty, closed and convex subset of $E$. Let $T: C \rightarrow E$ be a mapping satisfying the following;

$$
\phi(z, T x) \leq \phi(z, x), \quad \forall x \in C, z \in F(T) .
$$

Then $F(T)$ is closed and convex.

The following is our main result.

Theorem 3.1. Let $E$ be a uniformly convex and uniformly smooth Banach space which the duality mapping $J_{E}$ is weakly sequentially continuous. Let $C$ be a nonempty, closed and convex subset of $E$ such that $J_{E} C$ is closed and convex. Let $A \subset E \times E^{*}$ be a maximal monotone operator satisfying $D(A) \subset C$ and let $Q_{\mu}$ be a generalized resolvent of $A$, i.e., $Q_{\mu}=\left(J_{E}+\mu A\right)^{-1} J_{E}$ for all $\mu>0$. Let $T$ and $U$ be relatively nonexpansive mappings of $C$ into itself. Suppose that

$$
\Omega=F(T) \cap F(U) \cap A^{-1} 0 \neq \emptyset .
$$

For any $x_{1}=x \in C$, define $\left\{x_{n}\right\}$ as follows:

$$
\left\{\begin{array}{l}
y_{n}=J_{E}^{-1}\left(\left(1-r_{n}\right) J_{E} x_{n}+r_{n} J_{E} U Q_{\mu_{n}} x_{n}\right), \\
x_{n+1}=J_{E}^{-1}\left(\left(1-\beta_{n}\right) J_{E} x_{n}+\beta_{n} J_{E} T y_{n}\right), \quad \forall n \in \mathbb{N}
\end{array}\right.
$$

where $\left\{\mu_{n}\right\} \subset(0, \infty),\left\{\beta_{n}\right\} \subset(0,1), a, b, \delta, \gamma \in \mathbb{R}$ and $\left\{r_{n}\right\} \subset(0,1)$ satisfy the following:

$$
0<a \leq \beta_{n} \leq b<1,0<\delta \leq r_{n} \leq \gamma<1 \text { and } 0<c \leq \mu_{n}, \quad \forall n \in \mathbb{N} .
$$

Then the sequence $\left\{x_{n}\right\}$ converges weakly to an elementt $z_{0} \in \Omega$, where $z_{0}=\lim _{n \rightarrow \infty} \Pi_{\Omega} x_{n}$.

Proof. Since $T$ and $U$ are relatively nonexpansive, we have that $F(T)$ and $F(U)$ are closed and convex. Since $A$ is a maximal monotone operator, we have that $A^{-1} 0$ is closed and convex. It follows that $\Omega=F(T) \cap F(U) \cap A^{-1} 0$ is closed and convex. Let $z \in \Omega$. Then $z=Q_{\mu_{n}} z, z=T z$ and $z=U z$. Put

$$
y_{n}=J_{E}^{-1}\left(\left(1-r_{n}\right) J_{E} x_{n}+r_{n} J_{E} U Q_{\mu_{n}} x_{n}\right)
$$

and $z_{n}=Q_{\mu_{n}} x_{n}$ for all $n \in \mathbb{N}$. We have

$$
\begin{aligned}
\phi\left(z, y_{n}\right)= & \phi\left(z, J_{E}^{-1}\left(\left(1-r_{n}\right) J_{E} x_{n}+r_{n} J_{E} U z_{n}\right)\right) \\
= & \|z\|^{2}-2\left\langle z,\left(1-r_{n}\right) J_{E} x_{n}+r_{n} J_{E} U z_{n}\right\rangle \\
& +\left\|\left(1-r_{n}\right) J_{E} x_{n}+r_{n} J_{E} U z_{n}\right\|^{2} \\
\leq & \|z\|^{2}-2\left(1-r_{n}\right)\left\langle z, J_{E} x_{n}\right\rangle-2 r_{n}\left\langle z, J_{E} U z_{n}\right\rangle \\
& +\left(1-r_{n}\right)\left\|x_{n}\right\|^{2}+r_{n}\left\|U z_{n}\right\|^{2} \\
= & \left(1-r_{n}\right) \phi\left(z, x_{n}\right)+r_{n} \phi\left(z, U z_{n}\right) \\
\leq & \left(1-r_{n}\right) \phi\left(z, x_{n}\right)+r_{n} \phi\left(z, z_{n}\right) \\
\leq & \left(1-r_{n}\right) \phi\left(z, x_{n}\right)+r_{n} \phi\left(z, x_{n}\right) \\
= & \phi\left(z, x_{n}\right) .
\end{aligned}
$$


Similarly, we also have

$$
\begin{aligned}
\phi\left(z, x_{n+1}\right) & =\phi\left(z, J_{E}^{-1}\left(\left(1-\beta_{n}\right) J_{E} x_{n}+\beta_{n} J_{E} T y_{n}\right)\right) \\
& \leq\left(1-\beta_{n}\right) \phi\left(z, x_{n}\right)+\beta_{n} \phi\left(z, T y_{n}\right) \\
& \leq\left(1-\beta_{n}\right) \phi\left(z, x_{n}\right)+\beta_{n} \phi\left(z, y_{n}\right) \\
& \leq\left(1-\beta_{n}\right) \phi\left(z, x_{n}\right)+\beta_{n} \phi\left(z, x_{n}\right) \\
& =\phi\left(z, x_{n}\right) .
\end{aligned}
$$

Then $\lim _{n \rightarrow \infty} \phi\left(z, x_{n}\right)$ exists. Thus, $\left\{x_{n}\right\},\left\{U z_{n}\right\},\left\{y_{n}\right\}$ and $\left\{T y_{n}\right\}$ are bounded. Putting

$$
r=\max \left\{\sup _{n \in \mathbb{N}}\left\|J_{E} x_{n}\right\|, \sup _{n \in \mathbb{N}}\left\|J_{E} U z_{n}\right\|, \sup _{n \in \mathbb{N}}\left\|J_{E} T y_{n}\right\|\right\}
$$

we have from Lemma 2.3 that there exists a strictly increasing, continuous and convex function $g:[0, \infty) \rightarrow[0, \infty)$ such that $g(0)=0$ and

$$
\|\lambda x+(1-\lambda) y\|^{2} \leq \lambda\|x\|^{2}+(1-\lambda)\|y\|^{2}-\lambda(1-\lambda) g(\|x-y\|)
$$

for all $x, y \in B_{r}$ and $\lambda$ with $0 \leq \lambda \leq 1$, where $B_{r}=\left\{z \in E^{*}:\|z\| \leq r\right\}$. Using this, we have that, for $n \in \mathbb{N}$ and $z \in \Omega$,

$$
\begin{aligned}
\phi\left(z, y_{n}\right)= & \phi\left(z, J_{E}^{-1}\left(\left(1-r_{n}\right) J_{E} x_{n}+r_{n} J_{E} U z_{n}\right)\right) \\
= & \|z\|^{2}-2\left\langle z,\left(1-r_{n}\right) J_{E} x_{n}+r_{n} J_{E} U z_{n}\right\rangle+\left\|\left(1-r_{n}\right) J_{E} x_{n}+r_{n} J_{E} U z_{n}\right\|^{2} \\
\leq & \|z\|^{2}-2\left\langle z,\left(1-r_{n}\right) J_{E} x_{n}+r_{n} J_{E} U z_{n}\right\rangle \\
& +\left(1-r_{n}\right)\left\|x_{n}\right\|^{2}+r_{n}\left\|U z_{n}\right\|^{2}-r_{n}\left(1-r_{n}\right) g\left(\left\|J_{E} x_{n}-J_{E} U z_{n}\right\|\right) \\
= & \left(1-r_{n}\right) \phi\left(z, x_{n}\right)+r_{n} \phi\left(z, U z_{n}\right)-r_{n}\left(1-r_{n}\right) g\left(\left\|J_{E} x_{n}-J_{E} U z_{n}\right\|\right) \\
\leq & \left(1-r_{n}\right) \phi\left(z, x_{n}\right)+r_{n} \phi\left(z, z_{n}\right)-r_{n}\left(1-r_{n}\right) g\left(\left\|J_{E} x_{n}-J_{E} U z_{n}\right\|\right) \\
\leq & \phi\left(z, x_{n}\right)-r_{n}\left(1-r_{n}\right) g\left(\left\|J_{E} x_{n}-J_{E} U z_{n}\right\|\right) .
\end{aligned}
$$

Similarly, we have that

$$
\begin{aligned}
& \phi\left(z, x_{n+1}\right)=\phi\left(z, J_{E}^{-1}\left(\left(1-\beta_{n}\right) J_{E} x_{n}+\beta_{n} J_{E} T y_{n}\right)\right) \\
&=\|z\|^{2}-2\left\langle z,\left(1-\beta_{n}\right) J_{E} x_{n}+\beta_{n} J_{E} T y_{n}\right\rangle+\left\|\left(1-\beta_{n}\right) J_{E} x_{n}+\beta_{n} J_{E} T y_{n}\right\|^{2} \\
& \leq\|z\|^{2}-2\left\langle z,\left(1-\beta_{n}\right) J_{E} x_{n}+\beta_{n} J_{E} T y_{n}\right\rangle \\
&+\left(1-\beta_{n}\right)\left\|x_{n}\right\|^{2}+\beta_{n}\left\|T y_{n}\right\|^{2}-\beta_{n}\left(1-\beta_{n}\right) g\left(\left\|J_{E} x_{n}-J_{E} T y_{n}\right\|\right) \\
&=\left(1-\beta_{n}\right) \phi\left(z, x_{n}\right)+\beta_{n} \phi\left(z, T y_{n}\right)-\beta_{n}\left(1-\beta_{n}\right) g\left(\left\|J_{E} x_{n}-J_{E} T y_{n}\right\|\right) \\
& \leq\left(1-\beta_{n}\right) \phi\left(z, x_{n}\right)+\beta_{n} \phi\left(z, y_{n}\right)-\beta_{n}\left(1-\beta_{n}\right) g\left(\left\|J_{E} x_{n}-J_{E} T y_{n}\right\|\right) \\
& \leq\left(1-\beta_{n}\right) \phi\left(z, x_{n}\right)+\beta_{n}\left(\phi\left(z, x_{n}\right)-r_{n}\left(1-r_{n}\right) g\left(\left\|J_{E} x_{n}-J_{E} U z_{n}\right\|\right)\right) \\
&-\beta_{n}\left(1-\beta_{n}\right) g\left(\left\|J_{E} x_{n}-J_{E} T y_{n}\right\|\right) \\
&= \phi\left(z, x_{n}\right)-\beta_{n} r_{n}\left(1-r_{n}\right) g\left(\left\|J_{E} x_{n}-J_{E} U z_{n}\right\|\right)-\beta_{n}\left(1-\beta_{n}\right) g\left(\left\|J_{E} x_{n}-J_{E} T y_{n}\right\|\right) .
\end{aligned}
$$

Therefore, we have that

$$
\beta_{n}\left(1-\beta_{n}\right) g\left(\left\|J_{E} x_{n}-J_{E} T y_{n}\right\|\right) . \leq \phi\left(z, x_{n}\right)-\phi\left(z, x_{n+1}\right)
$$

and

$$
\beta_{n} r_{n}\left(1-r_{n}\right) g\left(\left\|J_{E} x_{n}-J_{E} U z_{n}\right\|\right) \leq \phi\left(z, x_{n}\right)-\phi\left(z, x_{n+1}\right) .
$$


We have from $0<a \leq \beta_{n} \leq b<1$ and $0<\delta \leq r_{n} \leq \gamma<1$ that

$$
\lim _{n \rightarrow \infty} g\left(\left\|J_{E} x_{n}-J_{E} T y_{n}\right\|\right)=\lim _{n \rightarrow \infty} g\left(\left\|J_{E} x_{n}-J_{E} U z_{n}\right\|\right)=0 .
$$

From the properties of $g$, we have that

$$
\lim _{n \rightarrow \infty}\left\|J_{E} x_{n}-J_{E} T y_{n}\right\|=\lim _{n \rightarrow \infty}\left\|J_{E} x_{n}-J_{E} U z_{n}\right\|=0 .
$$

From the definition of $y_{n}$, we also have that

$$
\left\|J_{E} x_{n}-J_{E} y_{n}\right\| \leq r_{n}\left\|J_{E} x_{n}-J_{E} U z_{n}\right\| .
$$

Since $\lim _{n \rightarrow \infty}\left\|J_{E} x_{n}-J_{E} U z_{n}\right\|=0$, we have $\left\|J_{E} x_{n}-J_{E} y_{n}\right\| \rightarrow 0$ and hence $\left\|J_{E} y_{n}-J_{E} T y_{n}\right\| \rightarrow 0$. Since $E^{*}$ is uniformly smooth, we have that

$$
\left\|y_{n}-T y_{n}\right\| \rightarrow 0 \text { and }\left\|x_{n}-U z_{n}\right\| \rightarrow 0
$$

as $n \rightarrow \infty$. Using $z_{n}=Q_{\mu_{n}} x_{n}$ and Lemma 2.6, we have that, for $z \in \Omega$,

$$
\phi\left(z_{n}, x_{n}\right)=\phi\left(Q_{\mu_{n}} x_{n}, x_{n}\right) \leq \phi\left(z, x_{n}\right)-\phi\left(z, Q_{\mu_{n}} x_{n}\right)=\phi\left(z, x_{n}\right)-\phi\left(z, z_{n}\right) .
$$

It follows from (3.1) that

$$
\begin{aligned}
\phi\left(z_{n}, x_{n}\right) & \leq \phi\left(z, x_{n}\right)-\phi\left(z, z_{n}\right) \\
& \leq \phi\left(z, x_{n}\right)-\frac{1}{r_{n}}\left(\phi\left(z, y_{n}\right)-\left(1-r_{n}\right) \phi\left(z, x_{n}\right)\right) \\
& =\frac{1}{r_{n}}\left(\phi\left(z, x_{n}\right)-\phi\left(z, y_{n}\right)\right) \\
& =\frac{1}{r_{n}}\left(\left\|x_{n}\right\|^{2}-\left\|y_{n}\right\|^{2}-2\left\langle z, J x_{n}-J y_{n}\right\rangle\right) \\
& \leq \frac{1}{r_{n}}\left(\left|\left\|x_{n}\right\|^{2}-\left\|y_{n}\right\|^{2}\right|+2\left|\left\langle z, J x_{n}-J y_{n}\right\rangle\right|\right) \\
& \leq \frac{1}{r_{n}}\left(\left|\left\|x_{n}\right\|-\left\|y_{n}\right\|\right|\left(\left\|x_{n}\right\|+\left\|y_{n}\right\|\right)+2\|z\|\left\|J x_{n}-J y_{n}\right\|\right) \\
& \leq \frac{1}{r_{n}}\left(\left\|x_{n}-y_{n}\right\|\left(\left\|x_{n}\right\|+\left\|y_{n}\right\|\right)+2\|z\|\left\|J x_{n}-J y_{n}\right\|\right) .
\end{aligned}
$$

This implies that $\lim _{n \rightarrow \infty} \phi\left(z_{n}, x_{n}\right)=0$. Since $E$ is uniformly convex and smooth, we have from Lemma 2.2 that

$$
\lim _{n \rightarrow \infty}\left\|z_{n}-x_{n}\right\|=0
$$

Since

$$
\left\|z_{n}-U z_{n}\right\| \leq\left\|z_{n}-x_{n}\right\|+\left\|x_{n}-U z_{n}\right\|
$$

we obtain that

$$
\lim _{n \rightarrow \infty}\left\|z_{n}-U z_{n}\right\|=0 \text {. }
$$

Since $\left\{x_{n}\right\}$ is bounded, there exists a subsequence $\left\{x_{n_{i}}\right\}$ of $\left\{x_{n}\right\}$ such that $x_{n_{i}} \rightarrow w$. From $\lim _{n \rightarrow \infty}\left\|x_{n}-y_{n}\right\|=0$ and $\lim _{n \rightarrow \infty}\left\|x_{n}-z_{n}\right\|=0$, we have $y_{n_{i}} \rightarrow w$ and $z_{n_{i}} \rightarrow w$. Using $\lim _{n \rightarrow \infty} \| z_{n}-$ $U z_{n} \|=0$ and the fact that $U$ is relatively nonexpansive, we have that $w=U w$ and hence $w \in F(U)$. Since $T$ is relatively nonexpansive, we have from $y_{n_{i}} \rightarrow w$ and $\left\|y_{n}-T y_{n}\right\| \rightarrow 0$ that $w \in F(T)$. This implies $w \in F(T) \cap F(U)$. 
Next, we show $w \in A^{-1} 0$. Since $J_{E}$ is uniformly norm-to-norm continuous on bounded sets, we conclude from (3.6)

$$
\lim _{n \rightarrow \infty}\left\|J_{E} x_{n}-J_{E} z_{n}\right\|=0
$$

From $\mu_{n} \geq c$, we have

$$
\lim _{n \rightarrow \infty} \frac{1}{\mu_{n}}\left\|J_{E} x_{n}-J_{E} z_{n}\right\|=0
$$

Therefore,

$$
\lim _{n \rightarrow \infty}\left\|B_{\mu_{n}} x_{n}\right\|=\lim _{n \rightarrow \infty} \frac{1}{\mu_{n}}\left\|J_{E} x_{n}-J_{E} z_{n}\right\|=0 .
$$

For $\left(p, p^{*}\right) \in A$, from the monotonicity of $A$, we have $\left\langle p-z_{n}, p^{*}-B_{\mu_{n}} x_{n}\right\rangle \geq 0$ for all $n \geq 0$. Replacing $n$ by $n_{i}$ and letting $i \rightarrow \infty$, we get $\left\langle p-w, p^{*}\right\rangle \geq 0$. From the maximallity of $A$, we have $w \in A^{-1} 0$. Therefore, $w \in \Omega$.

We next show that if $x_{n_{i}} \rightarrow u$ and $x_{n_{j}} \rightarrow v$, then $u=v$. In fact, we have that $u, v \in \Omega$. Put $a=\lim _{n \rightarrow \infty}\left(\phi\left(u, x_{n}\right)-\phi\left(v, x_{n}\right)\right)$. Since

$$
\phi\left(u, x_{n}\right)-\phi\left(v, x_{n}\right)=2\left\langle v-u, J_{E} x_{n}\right\rangle+\|u\|^{2}-\|v\|^{2}
$$

and the duality mapping $J_{E}$ of $E$ is weakly sequentially continuous, we have $a=2\left\langle v-u, J_{E} u\right\rangle+$ $\|u\|^{2}-\|v\|^{2}$ and $a=2\left\langle v-u, J_{E} v\right\rangle+\|u\|^{2}-\|v\|^{2}$. From these equalities, we obtain $2\langle v-u, J u-$ $J v\rangle=0$ and hence $\langle u-v, J u-J v\rangle=0$. From Lemma 2.1, it follows that $u=v$. Therefore, $\left\{x_{n}\right\}$ converges weakly to an element $z_{0} \in \Omega$.

Put $P=\Pi_{\Omega}$. We have from Lemma 2.5 and (3.2) that

$$
\begin{aligned}
\phi\left(P x_{n+1}, x_{n+1}\right) & \leq \phi\left(P x_{n+1}, x_{n+1}\right)+\phi\left(P x_{n}, P x_{n+1}\right) \\
& \leq \phi\left(P x_{n}, x_{n+1}\right) \\
& \leq \phi\left(P x_{n}, x_{n}\right)
\end{aligned}
$$

for all $n \in \mathbb{N}$. Hence $\lim _{n \rightarrow \infty} \phi\left(P x_{n}, x_{n}\right)$ exists. It follows from Lemma 2.5 that, for $k \in \mathbb{N}$,

$$
\begin{aligned}
\phi\left(P x_{n}, x_{n+k}\right)= & \phi\left(P x_{n}, P x_{n+k}\right)+\phi\left(P x_{n+k}, x_{n+k}\right) \\
& +2\left\langle P x_{n}-P x_{n+k}, J_{E} P x_{n+k}-J_{E} x_{n+k}\right\rangle \\
\geq & \phi\left(P x_{n}, P x_{n+k}\right)+\phi\left(P x_{n+k}, x_{n+k}\right)
\end{aligned}
$$

and hence

$$
\begin{aligned}
\phi\left(P x_{n}, P x_{n+k}\right) & \leq \phi\left(P x_{n}, x_{n+k}\right)-\phi\left(P x_{n+k}, x_{n+k}\right) \\
& \leq \phi\left(P x_{n}, x_{n}\right)-\phi\left(P x_{n+k}, x_{n+k}\right) .
\end{aligned}
$$

We also have from Lemma 2.5 that, for $p \in \Omega$,

$$
\phi\left(p, P x_{n}\right) \leq \phi\left(p, P x_{n}\right)+\phi\left(P x_{n}, x_{n}\right) \leq \phi\left(p, x_{n}\right) \leq \phi(p, x)
$$

and hence $\left\{P x_{n}\right\}$ is bounded. Using Lemma 2.4, we have that, for $m, n \in \mathbb{N}$ with $m>n$,

$$
g^{\prime}\left(\left\|P x_{n}-P x_{m}\right\|\right) \leq \phi\left(P x_{n}, P x_{m}\right) \leq \phi\left(P x_{n}, x_{n}\right)-\phi\left(P x_{m}, x_{m}\right),
$$

where $g^{\prime}$ is a strictly increasing, continuous and convex function such that $g^{\prime}(0)=0$. The the properties of $g^{\prime}$ yieeld that $\left\{P x_{n}\right\}$ ia a Cauchy sequence. Since $E$ is complete, $\left\{P x_{n}\right\}$ converges strongly to a point $u \in \Omega$. Furthermore, we have from Lemma 2.5 that

$$
\left\langle P x_{n}-z_{0}, J_{E} x_{n}-J_{E} P x_{n}\right\rangle \geq 0 .
$$


Since $x_{n} \rightarrow z_{0}$ and the duality mapping $J_{E}$ on $E$ is weakly sequentially continuous, we have that

$$
\left\langle u-z_{0}, J_{E} z_{0}-J_{E} u\right\rangle \geq 0
$$

and hence $\phi\left(u, z_{0}\right)+\phi\left(\left(z_{0}, u\right) \leq 0\right.$. This implies that $\phi\left(u, z_{0}\right)=\phi\left(z_{0}, u\right)=0$ and hence $u=z_{0}$. Therefore, $z_{0}=\lim _{n \rightarrow \infty} P x_{n}=\lim _{n \rightarrow \infty} \Pi_{\Omega} x_{n}$. This completes the proof.

\section{Applications}

In this section, using Theorem 3.1, we get well-known and new weak convergence theorems which are connected with relatively nonexpansive mappings and maximal monotone operators in Hilbert spaces and in Banach spaces. We first prove a weak convergence theorem for finding a zero point of a maximal monotone operator in a Banach space.

Theorem 4.1. Let $E$ be a uniformly convex and uniformly smooth Banach space which the duality mapping $J_{E}$ is weakly sequentially continuous. Let $A \subset E \times E^{*}$ be a maximal monotone operator and let $Q_{\mu}$ be a generalized resolvent of $A$, i.e., $Q_{\mu}=\left(J_{E}+\mu A\right)^{-1} J_{E}$ for all $\mu>0$. Suppose that $A^{-1} 0 \neq \emptyset$. For any $x_{1}=x \in E$, define $\left\{x_{n}\right\}$ as follows:

$$
x_{n+1}=J_{E}^{-1}\left(\left(1-r_{n}\right) J_{E} x_{n}+r_{n} J_{E} Q_{\mu_{n}} x_{n}\right),
$$

for all $n \in \mathbb{N}$, where $\left\{\mu_{n}\right\} \subset(0, \infty), \delta, \gamma \in \mathbb{R}$ and $\left\{r_{n}\right\} \subset(0,1)$ satisfy the following:

$$
0<\delta \leq r_{n} \leq \gamma<1 \text { and } 0<c \leq \mu_{n}, \quad \forall n \in \mathbb{N}
$$

Then the sequence $\left\{x_{n}\right\}$ converges weakly to an element $z_{0} \in A^{-1} 0$, where $z_{0}=\lim _{n \rightarrow \infty} \Pi_{A^{-1} 0} x_{n}$.

Proof. Putting $C=E$ and $T=U=I$ in Theorem 3.1, we obtain the desired result from Theorem 3.1.

Let $E$ be a Banach space and let $f: E \rightarrow(-\infty, \infty]$ be a proper, lower semicontinuous and convex function. Define the subdifferential of $f$ as follows:

$$
\partial f(x)=\left\{x^{*} \in E^{*}: f(y) \geq\left\langle y-x, x^{*}\right\rangle+f(x), \forall y \in E\right\}
$$

for all $x \in E$. Then we know that $\partial f$ is a maximal monotone operator; see [13] for more details. Let $E$ be a smooth, strictly convex and reflexive Banach space. Let $C$ be a nonempty, closed and convex subset of $E$. We have that there exists the generalized projection $\Pi_{C}$ of $E$ onto $C$. We also have that, for the indicator function $i_{C}$, that is,

$$
i_{C} x= \begin{cases}0, & x \in C, \\ \infty, & x \notin C,\end{cases}
$$


the subdifferential $\partial i_{C} \subset E \times E^{*}$ is a maximal monotone operator and the generalized resolvent $Q_{r}=\Pi_{C}$ of $\partial i_{C}$ for every $r>0$. In fact, for any $x \in E$ and $r>0$, we have that

$$
\begin{aligned}
z=Q_{r} x & \Leftrightarrow J_{E} z+r \partial i_{C} z \ni J_{E} x \\
& \Leftrightarrow J_{E} x-J_{E} z \in r \partial i_{C} z \\
& \Leftrightarrow i_{C} y \geq\left\langle y-z, \frac{J_{E} x-J_{E} z}{r}\right\rangle+i_{C} z, \forall y \in E \\
& \Leftrightarrow 0 \geq\left\langle y-z, J_{E} x-J_{E} z\right\rangle, \forall y \in C \\
& \Leftrightarrow z=\arg \min _{y \in C} \phi(y, x) \\
& \Leftrightarrow z=\Pi_{C} x .
\end{aligned}
$$

Using (4.1) and Theorem 3.1, we get the following weak convergence theorem for two relatively nonexpansive mappings in a Banach space.

Theorem 4.2. Let $E$ be a uniformly convex and uniformly smooth Banach space which the duality mapping $J_{E}$ is weakly sequentially continuous. Let $C$ be a nonempty, closed and convex subset of $E$ such that $J_{E} C$ is closed and convex. Let $T$ and $U$ be relatively nonexpansive mappings of $C$ into itself such that

$$
\Omega=F(T) \cap F(U) \neq \emptyset .
$$

For any $x_{1}=x \in C$, define $\left\{x_{n}\right\}$ as follows:

$$
\left\{\begin{array}{l}
y_{n}=J_{E}^{-1}\left(\left(1-r_{n}\right) J_{E} x_{n}+r_{n} J_{E} U x_{n}\right), \\
x_{n+1}=J_{E}^{-1}\left(\left(1-\beta_{n}\right) J_{E} x_{n}+\beta_{n} J_{E} T y_{n}\right), \quad \forall n \in \mathbb{N},
\end{array}\right.
$$

where $\left\{\beta_{n}\right\} \subset(0,1), a, b, \delta, \gamma \in \mathbb{R}$ and $\left\{r_{n}\right\} \subset(0,1)$ satisfy the following:

$$
0<a \leq \beta_{n} \leq b<1 \text { and } 0<\delta \leq r_{n} \leq \gamma<1, \quad \forall n \in \mathbb{N} .
$$

Then the sequence $\left\{x_{n}\right\}$ converges weakly to an element $z_{0} \in \Omega$, where $z_{0}=\lim _{n \rightarrow \infty} \Pi_{\Omega} x_{n}$.

Proof. Putting $A=\partial i_{C}$ in Theorem 3.1, we obtain that $Q_{\mu_{n}}=\Pi_{C}$ for all $\mu_{n}>0$. Therefore, we obtain the desired result from Theorem 3.1.

Let $H$ be a Hilbert space and let $C$ be a nonempty, closed and convex subset of $H$. A mapping $U: C \rightarrow H$ is called generalized hybrid [15] if there exist $\alpha, \beta \in \mathbb{R}$ such that

$$
\alpha\|U x-U y\|^{2}+(1-\alpha)\|x-U y\|^{2} \leq \beta\|U x-y\|^{2}+(1-\beta)\|x-y\|^{2}
$$

for all $x, y \in C$. Such a mapping $U$ is called $(\alpha, \beta)$-generalized hybrid. Notice that the class of $(\alpha, \beta)$-generalized hybrid mappings covers several well-known mappings. For example, a $(1,0)$-generalized hybrid mapping is nonexpansive. It is nonspreading $[12,16]$ for $\alpha=2$ and $\beta=1$, i.e.,

$$
2\|U x-U y\|^{2} \leq\|U x-y\|^{2}+\|U y-x\|^{2}, \quad \forall x, y \in C .
$$

It is also hybrid [17] for $\alpha=\frac{3}{2}$ and $\beta=\frac{1}{2}$, i.e.,

$$
3\|U x-U y\|^{2} \leq\|x-y\|^{2}+\|U x-y\|^{2}+\|U y-x\|^{2}, \quad \forall x, y \in C .
$$

In general, nonspreading and hybrid mappings are not continuous; see [14]. We know the following result obtained by Kocourek, Takahashi and Yao [15]; see also [18]. 
Lemma 4.1 ([15, 18]). Let $H$ be a Hilbert space, let $C$ be a nonempty, closed and convex subset of $H$ and let $U: C \rightarrow H$ be generalized hybrid. If $x_{n} \rightarrow z$ and $x_{n}-U x_{n} \rightarrow 0$, then $z \in F(U)$.

The following are two weak convergence theorems for finding a common element of the fixed point sets of two nonlinear operators and the zero point set of a maximal monotone operator in a Hilbert space.

Theorem 4.3. Let $H$ bea Hilbert space and let $C$ be a nonempty, closed and convex subset of $H$. Let $A \subset H \times H$ be a maximal monotone operator satisfying $D(A) \subset C$ and let $J_{\mu}$ be the resolvent of $A$, i.e., $J_{\mu}=(I+\mu A)^{-1}$ for all $\mu>0$. Let $T: C \rightarrow C$ be a nonspreading mapping and let $U: C \rightarrow C$ be a hybrid mapping. Suppose that $\Omega=F(T) \cap F(U) \cap A^{-1} 0 \neq \emptyset$. For any $x_{1}=x \in C$, define $\left\{x_{n}\right\}$ as follows:

$$
\left\{\begin{array}{l}
y_{n}=\left(1-r_{n}\right) x_{n}+r_{n} U J_{\mu_{n}} x_{n}, \\
x_{n+1}=\left(1-\beta_{n}\right) x_{n}+\beta_{n} T y_{n}, \quad \forall n \in \mathbb{N},
\end{array}\right.
$$

where $\left\{\mu_{n}\right\} \subset(0, \infty),\left\{\beta_{n}\right\} \subset(0,1), a, b, \delta, \gamma \in \mathbb{R}$ and $\left\{r_{n}\right\} \subset(0,1)$ satisfy the following:

$$
0<a \leq \beta_{n} \leq b<1,0<\delta \leq r_{n} \leq \gamma<1 \text { and } 0<c \leq \mu_{n}, \quad \forall n \in \mathbb{N} .
$$

Then the sequence $\left\{x_{n}\right\}$ converges weakly to an element $z_{0} \in \Omega$, where $z_{0}=\lim _{n \rightarrow \infty} P_{\Omega} x_{n}$ anf $P_{\Omega}$ is the metric projection of $H$ onto $\Omega$.

Proof. Since $T$ is nonspreading of $C$ into $C$, it satisfies the following:

$$
2\|T x-T y\|^{2} \leq\|T x-y\|^{2}+\|T y-x\|^{2}, \quad \forall x, y \in C .
$$

Putting $y=p$ for $p \in F(T)$, we have that

$$
2\|T x-p\|^{2} \leq\|T x-p\|^{2}+\|p-x\|^{2}, \quad \forall x \in C
$$

and hence

$$
\|T x-p\|^{2} \leq\|p-x\|^{2}, \quad \forall x \in C .
$$

This implies that $T$ is quasi-nonexpansive. Furthermore, we have from Lemma 4.1 that $T$ is demiclosed.

Similarly, since $U$ is a hybrid mapping of $C$ into $C$ such that $F(U) \neq \emptyset$, it satisfies the following:

$$
3\|U x-U y\|^{2} \leq\|x-y\|^{2}+\|U x-y\|^{2}+\|U y-x\|^{2}, \quad \forall x, y \in C .
$$

Putting $y=p$ for $p \in F(U)$, we have that

$$
3\|U x-p\|^{2} \leq\|x-p\|^{2}+\|U x-p\|^{2}+\|p-x\|^{2}, \quad \forall x \in C
$$

and hence

$$
\|U x-p\|^{2} \leq\|p-x\|^{2}, \quad \forall x \in C .
$$

This implies that $U$ is quasi-nonexpansive. Furthermore, we have from Lemma 4.1 that $U$ is demiclosed. Therefore, we have the desired result from Theorem 3.1.

Theorem 4.4. Let $H$ bea Hilbert space and let $C$ be a nonempty, closed and convex subset of $H$. Let $A \subset H \times H$ be a maximal monotone operator satisfying $D(A) \subset C$ and let $J_{\mu}$ be the resolvent of $A$, i.e., $J_{\mu}=(I+\mu A)^{-1}$ for all $\mu>0$. Let $T: C \rightarrow C$ be a nonspreading mapping and let $U: C \rightarrow C$ be a generalized hybrid mapping. Suppose that

$$
\Omega=F(T) \cap F(U) \cap A^{-1} 0 \neq \emptyset .
$$


For any $x_{1}=x \in C$, define $\left\{x_{n}\right\}$ as follows:

$$
\left\{\begin{array}{l}
y_{n}=\left(1-r_{n}\right) x_{n}+r_{n} U J_{\mu_{n}} x_{n}, \\
x_{n+1}=\left(1-\beta_{n}\right) x_{n}+\beta_{n} T y_{n}, \quad \forall n \in \mathbb{N},
\end{array}\right.
$$

where $\left\{\mu_{n}\right\} \subset(0, \infty),\left\{\beta_{n}\right\} \subset(0,1), a, b, \delta, \gamma \in \mathbb{R}$ and $\left\{r_{n}\right\} \subset(0,1)$ satisfy the following:

$$
0<a \leq \beta_{n} \leq b<1,0<\delta \leq r_{n} \leq \gamma<1 \text { and } 0<c \leq \mu_{n}, \quad \forall n \in \mathbb{N} .
$$

Then the sequence $\left\{x_{n}\right\}$ converges weakly to an element $z_{0} \in \Omega$, where $z_{0}=\lim _{n \rightarrow \infty} P_{\Omega} x_{n}$ anf $P_{\Omega}$ is the metric projection of $H$ onto $\Omega$.

Proof. Since $T$ is a nonexpansive mapping of $C$ into $C$ with $F(T) \neq \emptyset$, we have that $T$ is quasinonexpansive. Furthermore, we have from Lemma 4.1 that $T$ is demiclosed. Since $U$ is a generalized hybrid mapping of $C$ into $C$ such that $F(U) \neq \emptyset, U$ is quasi-nonexpansive. Furthermore, from Lemma 4.1, $U$ is demiclosed. Therefore, we have the desired result from Theorem 3.1 .

The following is a weak convergence theorems for finding a common point of three sets in a Banach space.

Theorem 4.5. Let $E$ be a uniformly convex and uniformly smooth Banach space which the duality mapping $J_{E}$ of $E$ is weakly suquentially continuous. Let $C, D$ and $F$ be nonempty, closed and convex subsets of $E$. Let $\Pi_{C}, \Pi_{D}$ and $\Pi_{F}$ be the generalized projections of $E$ onto $C, D$ and $F$, respectively. Suppose that $C \cap D \cap F \neq \emptyset$. For any $x_{1}=x \in E$, define

$$
\left\{\begin{array}{l}
\left.y_{n}=J_{E}^{-1}\left(\left(1-r_{n}\right) J_{E} x_{n}-+r_{n} J_{E} \Pi_{D} \Pi_{F} x_{n}\right)\right), \\
x_{n+1}=J_{E}^{-1}\left(\left(1-\beta_{n}\right) J_{E} x_{n}+\beta_{n} J_{E} \Pi_{C} y_{n}\right), \quad \forall n \in \mathbb{N},
\end{array}\right.
$$

where $\left\{\beta_{n}\right\} \subset(0,1)$ and $\left\{r_{n}\right\} \subset(0,1)$ satisfy the following:

$$
0<a \leq \beta_{n} \leq b<1 \text { and } 0<\delta \leq r_{n} \leq \gamma<1, \quad \forall n \in \mathbb{N}
$$

for some $a, b, \delta, \gamma \in \mathbb{R}$. Then $\left\{x_{n}\right\}$ converges weakly to $z_{0} \in C \cap D \cap F$. where $z_{0}=\lim _{n \rightarrow \infty} \Pi_{C \cap D \cap F} x_{n}$.

Proof. Take $A=\partial i_{F}$ in Theorem 3.1. Then we have that $Q_{\mu_{n}}=\Pi_{F}$ for all $n \in \mathbb{N}$. Furthermore, since $\Pi_{C}$ is the genralized projection of $E$ onto $C$, we have from Lemma 2.5 that

$$
\phi\left(z, \Pi_{C} x\right) \leq \phi(z, x), \quad \forall x \in E, z \in C .
$$

We show that $\Pi_{C}$ is demiclosed. In fact, assume that $x_{n} \rightarrow p$ and $x_{n}-\Pi_{C} x_{n} \rightarrow 0$. It is clear that $\Pi_{C} x_{n} \rightarrow p$. Since $E$ is uniformly smooth, we have that $\left\|J_{E} x_{n}-J_{E} \Pi_{C} x_{n}\right\| \rightarrow 0$. Since $\Pi_{C}$ is the generalized projection of $E$ onto $C$, we have that

$$
\left\langle\Pi_{C} x_{n}-\Pi_{C} p, J_{E} x_{n}-J_{E} \Pi_{C} x_{n}-\left(J_{E} p-J_{E} \Pi_{C} p\right)\right\rangle \geq 0 .
$$

Therefore, $\left\langle p-\Pi_{C} p,-\left(J_{E} p-J_{E} \Pi_{C} p\right)\right\rangle \geq 0$ and hence $\phi\left(p, \Pi_{C} p\right)+\phi\left(\Pi_{C} p, p\right) \leq 0$. This implies that $p=\Pi_{C} p$ and hence $\Pi_{C}$ is demiclosed. Similarly,

$$
\phi\left(z, \Pi_{D} x\right) \leq \phi(z, x), \quad \forall x \in E, z \in D
$$

and $\Pi_{D}$ is demiclosed. Therefore, we have the desired result from Theorem 3.1.

The following is a weak convergence theorem for finding a common element of zero point sets of three maximal monotone operators of a Banach space. 
Theorem 4.6. Let $E$ be a uniformly convex and uniformly smooth Banach space which the duality mapping $J_{E}$ of $E$ is weakly suquentially continuous. Let $A, B$ and $G$ be maximal monotone operators of $E$ into $E^{*}$. Let $Q_{r}^{A}$ be the generalized resolvent of $A$ for $r>0, Q_{\mu}^{B}$ be the generalized resolvent of $B$ for $\mu>0$ and let $Q_{\lambda}^{G}$ be the generalized resolvent of $G$ for $\lambda>0$. Suppose that

$$
\Omega=A^{-1} 0 \cap B^{-1} 0 \cap G^{-1} 0 \neq \emptyset .
$$

For any $x_{1}=x \in E$, define

$$
\left\{\begin{array}{l}
\left.y_{n}=J_{E}^{-1}\left(\left(1-r_{n}\right) J_{E} x_{n}+r_{n} Q_{\lambda}^{G} Q_{r}^{A} x_{n}\right)\right), \\
x_{n+1}=J_{E}^{-1}\left(\left(1-\beta_{n}\right) J_{E} x_{n}+\beta_{n} J_{E} Q_{\mu}^{B} y_{n}\right), \quad \forall n \in \mathbb{N},
\end{array}\right.
$$

where $\left\{\beta_{n}\right\} \subset(0,1)$ and $\left\{r_{n}\right\} \subset(0,1)$ satisfy the following:

$$
0<a \leq \beta_{n} \leq b<1 \text { and } 0<\delta \leq r_{n} \leq \gamma<1, \quad \forall n \in \mathbb{N}
$$

for some $a, b, \delta, \gamma \in \mathbb{R}$. Then the sequence $\left\{x_{n}\right\}$ converges weakly to a point $z_{0} \in \Omega$. where $z_{0}=\lim _{n \rightarrow \infty} \Pi_{\Omega} x_{n}$.

Proof. Take $\mu_{n}=r$ for $r>0$ in Theorem 3.1. Then we have that $Q_{\mu_{n}}^{A}=Q_{r}^{A}$ for all $n \in \mathbb{N}$. Furthermore, since $Q_{\mu}^{B}$ is the generalized resolvent of $B$, we have from Lemma 2.6 that

$$
\phi\left(z, Q_{\mu}^{B} x\right) \leq \phi(z, x), \quad \forall x \in E, z \in B^{-1} 0 .
$$

Next, we show that $Q_{\mu}^{B}$ is demiclosed. In fact, assume that $x_{n} \rightarrow p$ and $x_{n}-Q_{\mu}^{B} x_{n} \rightarrow 0$. It is clear that $Q_{\mu}^{B} x_{n} \rightarrow p$ as $n \rightarrow \infty$. Since $E$ is unifrmly smooth, we have that $\left\|J_{E} x_{n}-J_{E} Q_{\mu}^{B} x_{n}\right\| \rightarrow 0$. Since $Q_{\mu}^{B}$ is the generalized resolvent of $B$, we have from [19] that

$$
\left\langle Q_{\mu}^{B} x_{n}-Q_{\mu}^{B} p, J_{E} x_{n}-J_{E} Q_{\mu}^{B} x_{n}-\left(J_{E} p-J_{E} Q_{\mu}^{B} p\right)\right\rangle \geq 0 .
$$

Therefore, $\left\langle p-Q_{\mu}^{B} p,-\left(J_{F} p-J_{E} Q_{\mu}^{B} p\right)\right\rangle \geq 0$ and hence $\phi\left(p, Q_{\mu}^{B} p\right)+\phi\left(Q_{\mu}^{B} p, p\right) \leq 0$. This implies that $p=Q_{\mu}^{B} p$ and hence $Q_{\mu}^{B}$ is demiclosed.

Similarly,

$$
\phi\left(z, Q_{\lambda}^{G} x\right) \leq \phi(z, x), \quad \forall x \in E, z \in G^{-1} 0
$$

and $Q_{\lambda}^{G}$ is demiclosed. Therefore, we have from Theorem 3.1 the desired result immediately.

\section{REFERENCES}

[1] S. Matsushita, W. Takahashi, A strong convergence theorem for relatively nonexpansive mappings in a Banach space, J. Approx. Theory 134 (2005), 257-266.

[2] W. R. Mann, Mean value methods in iteration, Proc. Amer. Math. Soc. 4 (1953), 506-510.

[3] S. Reich, Weak convergence theorems for nonexpansive mappings in Banach spaces, J. Math. Anal. Appl. 67 (1979), 274-276.

[4] S. Matsushita, W. Takahashi, Weak and strong convergence theorems for relatively nonexpansive mappings in Banach spaces, Fixed Point Theory Appl. 2004 (2004), 37-47.

[5] W. Takahashi, Introduction to Nonlinear and Convex Analysis, Yokohama Publishers, Yokohama, 2009.

[6] W. Takahashi, Nonlinear Functional Analysis, Yokohama Publishers, Yokohama, 2000.

[7] W. Takahashi, Convex Analysis and Approximation of Fixed Points, Yokohama Publishers, Yokohama, 2000.

[8] Y. I. Alber, Metric and generalized projections in Banach spaces: Properties and applications, in Theory and Applications of Nonlinear Operators of Accretive and Monotone Type, A. G. Kartsatos (Ed.), Marcel Dekker, New York, 1996, pp. 15-50, 
[9] S. Kamimura, W. Takahashi, Strong convergence of a proximal-type algorithm in a Banach space, SIAM. J. Optim. 13 (2002), 938-945.

[10] H. K. Xu, Another control condition in an iterative method for nonexpansive mappings, Bull. Austral. Math. Soc. 65 (2002), 109-113.

[11] F. E. Browder, Nonlinear maximal monotone operators in Banach spaces, Math. Ann. 175 (1968), 89-113.

[12] F. Kohsaka, W. Takahashi, Existence and approximation of fixed points of firmly nonexpansive-type mappings in Banach spaces, SIAM. J. Optim. 19 (2008), 824-835.

[13] R. T. Rockafellar, On the maximal monotonicity of subdifferential mappings, Pacific J. Math. 33 (1970), 209-216.

[14] T. Igarashi, W. Takahashi, K. Tanaka, Weak convergence theorems for nonspreading mappings and equilibrium problems, in Nonlinear Analysis and Optimization (S. Akashi, W. Takahashi and T. Tanaka Eds.), pp. 75-85, Yokohama Publishers, Yokohama, 2008.

[15] P. Kocourek, W. Takahashi, J.-C. Yao, Fixed point theorems and weak convergence theorems for generalized hybrid mappings in Hilbert space, Taiwanese J. Math. 14 (2010), 2497-2511.

[16] F. Kohsaka, W. Takahashi, Fixed point theorems for a class of nonlinear mappings related to maximal monotone operators in Banach spaces, Arch. Math. 91 (2008), 166-177.

[17] W. Takahashi, Fixed point theorems for new nonlinear mappings in a Hilbert space, J. Nonlinear Convex Anal. 11 (2010), 79-88.

[18] W. Takahashi, J.-C. Yao, P. Kocourek, Weak and strong convergence theorems for generalized hybrid nonselfmappings in Hilbert spaces, J. Nonlinear Convex Anal. 11 (2010), 567-586.

[19] K. Aoyama, F. Kohsaka, W. Takahashi, Three generalizations of firmly nonexpansive mappings: Their relations and continuous properties, J. Nonlinear Convex Anal. 10 (2009), 131-147. 